\title{
The Fast Interlock system of W7-X - first experience
}

\author{
Reinhard Vilbrandt, Jörg Schacht, Uwe Herbst, Stefan Marsen, Kian Rahbarnia, Kai Jakob Brunner, \\ Matthias Hirsch, Jens Knauer, Dirk Naujoks, Sven Degenkolbe, Hans-Stephan Bosch, W7-X Team
}

\author{
Max-Planck-Institute for Plasma Physics, D-17491 Greifswald, Germany
}

\begin{abstract}
This paper focuses on the design, installation and test of the first version of the Fast Interlock System (FIS) of W7-X. The structure of the system and the basic components including some safety-relevant plasma diagnostics are described. The very first practical experiences with the FIS have been gained during the operation phase $1.2 \mathrm{~b}$ in summer 2018. Next to the proof of a satisfactory reaction, scalability and operability, the focus also was on the assessment of the reaction times of the core system and the various safety-relevant plasma diagnostics during several experimental situations. A couple of planned measures to improve and extend the FIS will be presented in this paper.
\end{abstract}

Keywords: Protection, Interlock system, W7-X, Risk assessment, Plasma

\section{Introduction}

The safety related programmable logic controller (PLC) in the central safety system (cSS) of W7-X with its corresponding periphery ensures occupational safety and the basic protection of investment. Despite its high reliability the response time is moderate and in the range of some $100 \mathrm{~ms}$ only. The cSS is not sufficient to protect components in the plasma vessel from rapid overload by the plasma itself or from direct loads resulting from the heating systems. Therefore the additional Fast Interlock System (FIS) is required which can respond to the signals of any safety-relevant diagnostics within significantly shorter time.

In this context, the FIS must be considered as an integrated part of the safety system together with the cSS. The FIS has properties of functional safety concerning the device protection. Design and realization along IEC 61511 with the application of a common Project Safety Plan [1] offers the opportunity to complement the safety instrumented functions (SIF) of the cSS by very fast functions of the FIS to close the gap in safety, or to mitigate the risks of disastrous damages, although the confirmation of the realized SIL for the FIS is often rather difficult.[2] [3]

\section{General design and structure of the FIS}

Three systems are used for the heating of the plasma inside of W7-X, namely the Electron Cyclotron Resonance Heating $(\mathrm{ECRH})$ with $7.5 \mathrm{MW}$ heating power (ultimately up to $15 \mathrm{MW}$ ), the Neutral Beam Injection Heating (NBI) - 1.7 MW (in the future up to $10 \mathrm{MW}$ ), and the Ion Cyclotron Resonance Heating (ICRH), which shall be available as from 2021. The enormous energy fluxes produced by these systems and the energy deposited in the plasma itself endanger mainly the components in the plasma vessel and in the end the mechanical integrity of the machine. To switch off the heating systems fast enough is essential, if the thermal loads of components in the plasma vessel get too large.
The calculations carried out concluded that the complete reaction time of the FIS must be at most $100 \mathrm{~ms}$, from the measurement to the fast switch-off of the heating power.

The response time of the central FIS (cFIS) was limited to about $1 \mathrm{~ms}$ intentionally, to ensure a measurement time as long as possible for safety-relevant diagnostics, which mainly consist of special developed instruments. They must deliver reliable signals, which indicate forbidden loads or plasma states.

The FIS (Fig. 1) is a distributed system with the central Fast Interlock System (cFIS) as the central component, several local FIS at each heating system, and the so-called safety-relevant diagnostic instruments (diagnostics).

To reduce the complexity of the FIS the cFIS contains only the necessary functions for the supervision of plasma operations. The functions to the safe operation of the heating systems themselves, which are also used for internal tests and conditioning modes, were realized in the local FIS.

The safety signals sent from the diagnostics to the cFIS and the high voltage power supplies were restricted to Boolean ones. A programmable Boolean processor is the heart of the cFIS.

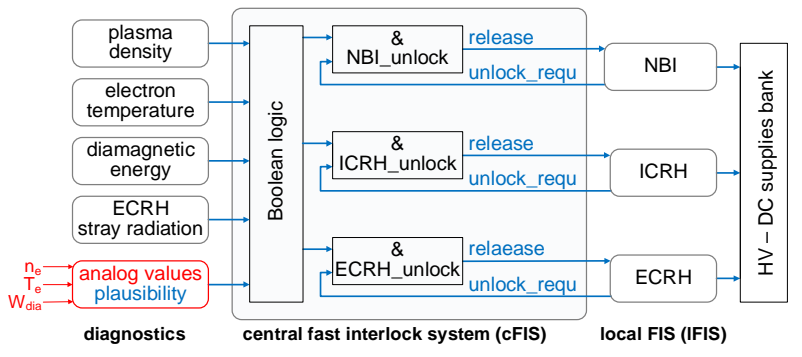

Fig. 1 the first Fast Interlock System before OP1.2 (the plausibility test has not been implemented yet)

All safety-relevant signals must be active signals in the sense of fail-safe principle. That means also, each heating system must send a request_signal to the cFIS; 
and it can switch on the assigned power supply only if the release_signal is active. The release_signal is set to active state only if no forbidden state is detected.

The signal converters in the field must be robust against magnetic disturbances.

\subsection{The central fast interlock system}

The cFIS hardware consists of industrially available components only (Fig. 2). The complete processing cycle of the high-speed Boolean processor SIMATIC S7-300, FM352-5 (12 DI / 8 DO) is in the range of $1 \mu \mathrm{s}$. Ladder Diagram (LD) (IEC 61131) is used as programming tool.

An S7-400 (with STEP 7) with a cycle time of $10 \mathrm{~ms}$ is used for cFIS-operator interactions and the control of the Boolean processor.

It is possible to preset values, e.g. limits, thresholds, or measurement intervals of the diagnostics via LANconnection. The parameter setting concept of a simple write and read-back algorithm to check the correctness of communication was found to be safe and convenient.

The Interlock signal receiver and transmitter modules EKS-IOL-3000 (eks Engel FOS GmbH \& Co. KG) with optical links were tested before to guarantee their functionality in stationary magnet fields up to $30 \mathrm{mT}$. The resulting high transmission time of $400 \mu$ s can be tolerated.

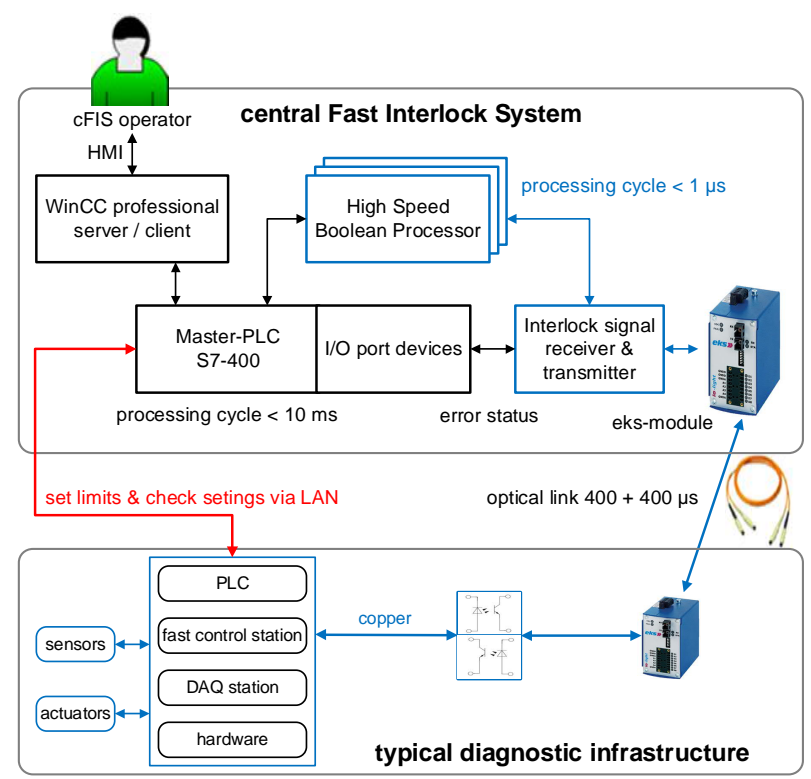

Fig. 2 The central fast interlock system (cFIS)

\subsection{Local fast interlock systems}

Each heating system hosts its own local fast interlock system, which is directly connected to the selected high voltage supply by safe hardware plugs. In this way, the uniform high voltage power supplies of W7 X can be assigned to the individual heating systems temporarily without the control being lost.

Blocking of the insulated-gate bipolar transistor (IGBT) in the high voltage modules is used uniformly as the fastest way to switch off the heating power. Switching times of about $5 \mu$ s can be reached here.

The different heating systems have special diagnostics for safe operation, e.g. the sniffer probes at the ECRH.
These special diagnostics remain assigned to the local fast interlock systems. Only if the signals are of primary importance, a Boolean signal is generated and and sent to the cFIS.

\subsubsection{The local FIS of the ECRH}

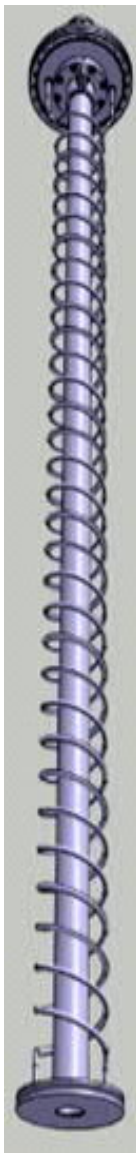

The first safety-relevant diagnostic, which was available at W7-X, was the measurement of the ECRH stray radiation to detect an insufficient absorption of the beam energy by the plasma. This measurement is performed with five stray radiation monitors distributed around the machine. The detector diode receives any incoming $\mu$-wave radiation, which is guided from the plasma vessel by open oversized copper tubes and collected by a receiver optic that provides broad antenna pattern (Fig. 3). [5]

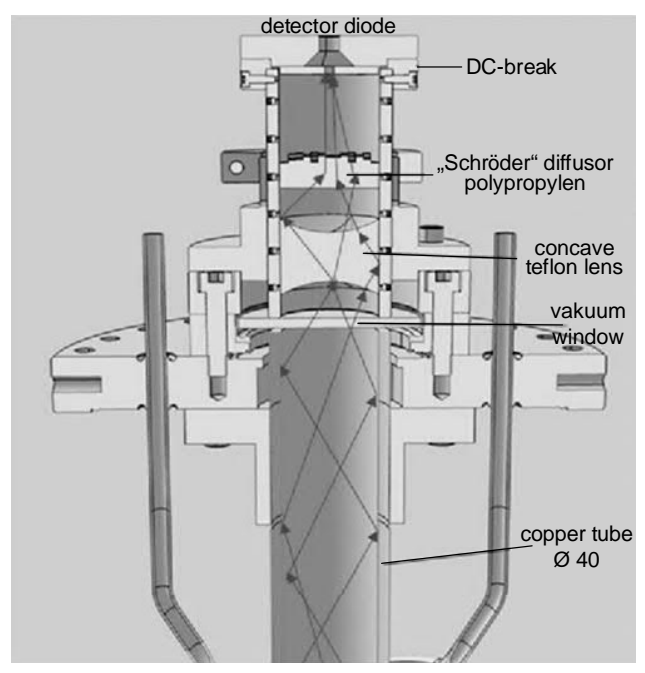

Fig. 3 ECRH-stray radiation monitor (guiding tube and optic)

The so-called sniffer probes measures both the radiation in the ECRH beamdump and the radiation in the vessel. The increased stray radiation level during the plasma build-up phase $(\sim 50-500 \mathrm{~ms})$ is used as a test of the functionality of the diagnostic in the beginning of each experiment.

Because a low plasma density (almost always) is the reason for an increased radiation, this signal also is sent to the cFIS to switch off all heating systems (Fig. 4). The cFIS operator can set the allowed stray radiation level for the beamdump and the plasma vessel by setting of the integration time.

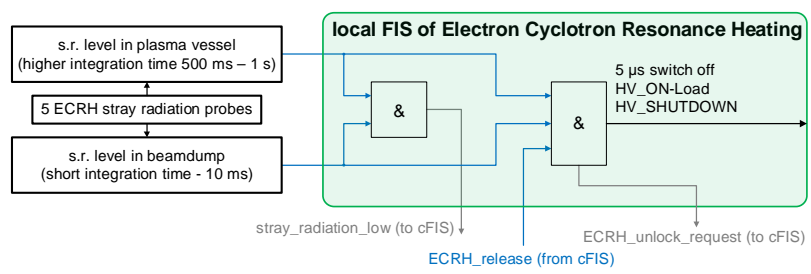

Fig. 4 local FIS of the ECRH

\subsubsection{The local FIS of the NBI}

In the local FIS of the NBI (Fig. 6) the heat shield thermography (HST) observes the surface temperature of the carbon target tiles for the two active NBI sources across the AET21 port [4] (Fig. 5). This shall protect the 
tiles from overheating at e.g. bad absorption of the energy in the plasma. A daily test of HST with reduced power (no plasma) is carried out as well as a self-test of the pyrometer before each NBI-radiation into the plasma for reliability reasons. The limit for the tile temperature was set to $\mathrm{T}_{\max } \leq 1,000{ }^{\circ} \mathrm{C}$.

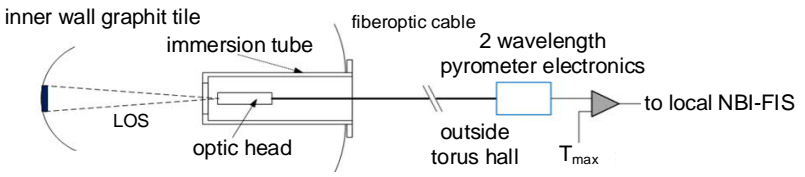

Fig. 5 heat shield thermography of the NBI

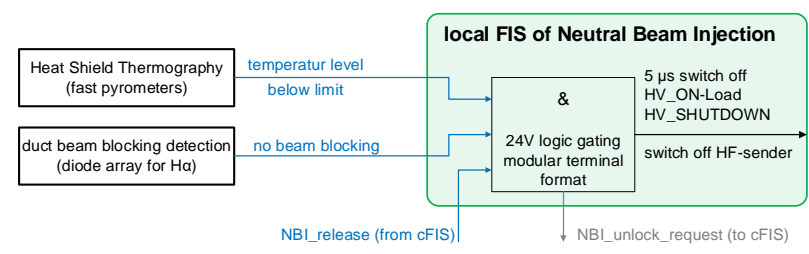

Fig. 6 local FIS of the NBI

\subsection{Other safety-relevant diagnostics}

\subsubsection{Plasma density}

The Single Channel (dispersion) interferometer (Fig. 7) measures the plasma density. By using of 2 frequency doubling crystals the final phase shift at the detector is insensitive to geometrical changes of the beam path length, e.g. due to vibrations. [6]

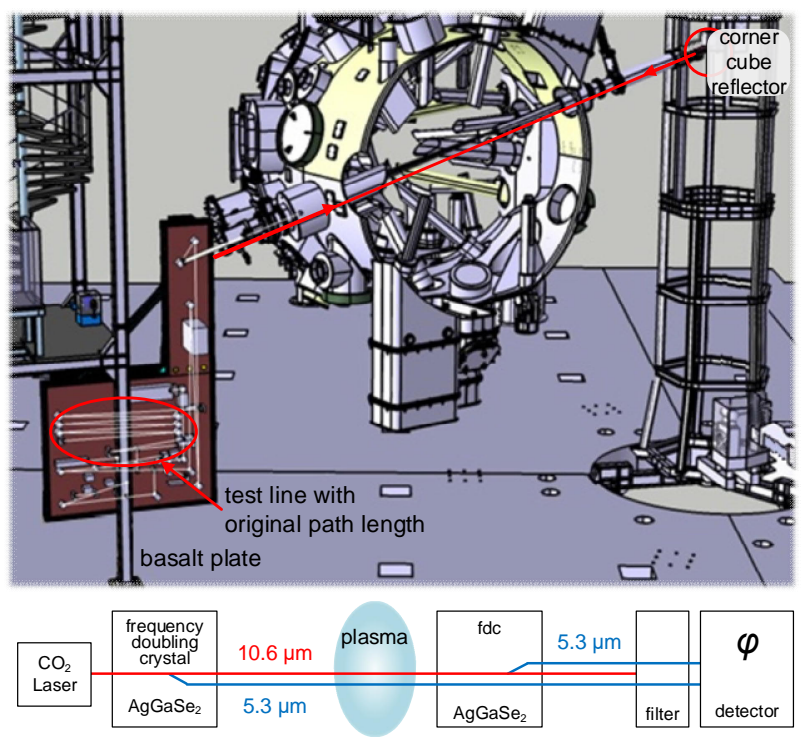

Fig. 7 The single channel interferometer in W7-X

It delivers Boolean signals for several plasma densities $\mathrm{n}_{\text {min-NBI }}$ for allowing NBI operation, $\mathrm{n}_{\text {cut-off }}$ for ECE operation, and $\mathrm{n}_{\min }$ for sufficient plasma. The cFIS operator can set these thresholds before plasma discharges separately.

\subsubsection{Electron temperature}

The Electron Cyclotron Emission (ECE) (Fig. 8) delivers the electron temperature $\mathrm{T}_{\mathrm{e}}$, but only up to the

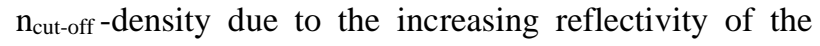
plasma. [7]

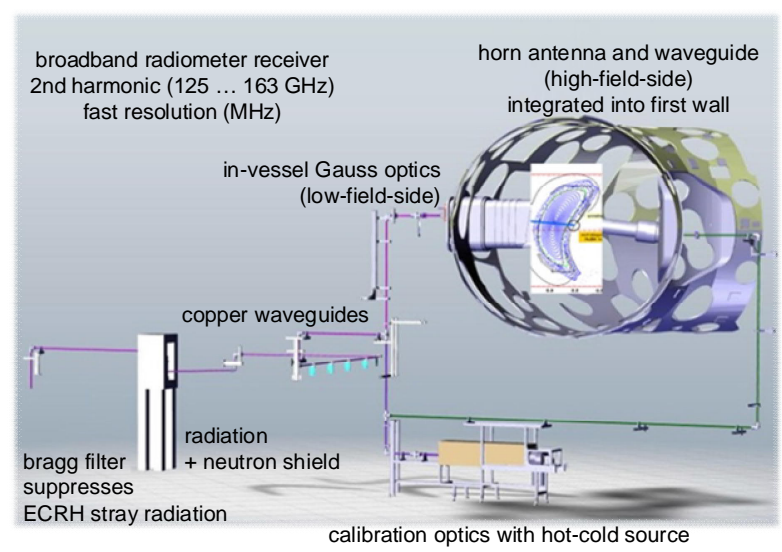

Fig. 8 ECE-measuring at W7-X

\subsubsection{Diamagnetic energy}

Three Diamagnetic loops (Fig. 9) are located inside the plasma vessel; 1 in triangular and 2 close to beanshaped plane to measure the diamagnetic energy inside the plasma. [8] [9]

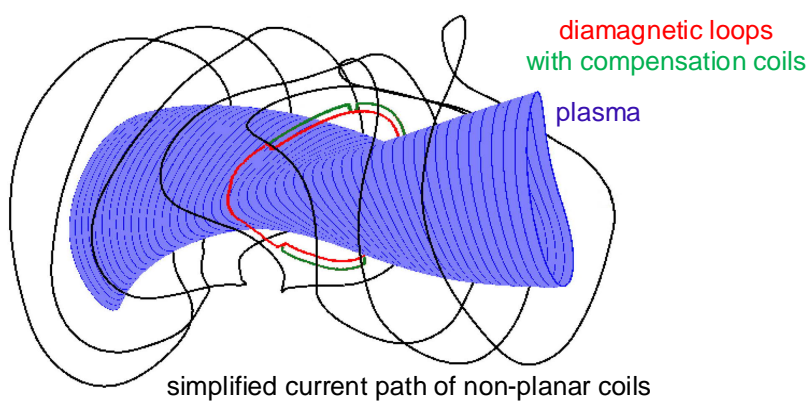

Fig. 9 Diamagnetic loop installed in W7-X

\subsubsection{The composed status "sufficient plasma"}

This is a redundant signal to the direct mesuaring diagnostics to increase the reliability.

While the HST measures a temperature and the sniffer probes measure a thermal load, the other (density, electron temperature, diamagnetic energy) do not measure the impact of dangerous energetic flows directly.

They are used inside the cFIS to compose a status called "sufficient plasma" (Fig. 10), which characterizes the properties of the real plasma allowing a safe operation without overloading the components. The heating systems are switched off in the case of an insufficient plasma to avoid an unnecessary load, although no direct measured signal indicates a dangerous situation.

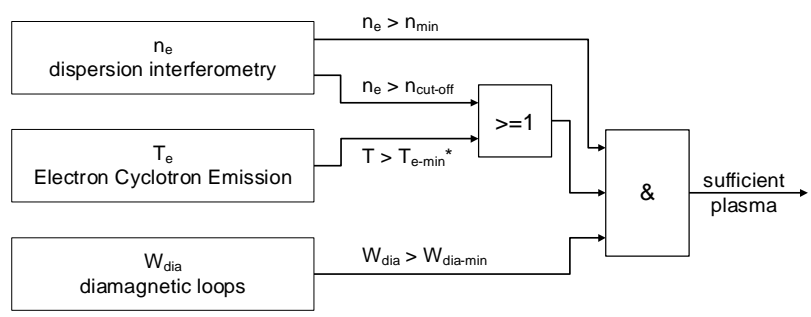

Fig. 10 generation of the status "sufficient plasma"

The parameters of "sufficient plasma" can be modified by the cFIS operator according to the gained experience with the plasma behavior for the following experiments step by step. 
The start values for OP1.2b operation were set according to the experience of previous discharges to

$\mathrm{n}_{\min } \geq 0.5 *-10^{19} \mathrm{~m}^{-3}$,

$\mathrm{T}_{\mathrm{e}-\mathrm{min}} \geq 0.5 \mathrm{keV}$,

$\mathrm{W}_{\mathrm{dia}} \geq 30 \mathrm{~kJ}$.

Additional precautions for this first test of the device were implemented, e.g. a minimum density for the NBI operation $\left(\mathrm{n}_{\min -\mathrm{NBI}} \geq 1.0 * 10^{19} \mathrm{~m}^{-3}\right)$ and some time constraints for heating duration.

\section{First experience}

The first practical experiences with the cFIS were gained during operation phase $1.2 \mathrm{~b}$ in summer 2018 . Because W7-X was equipped with an inertial cooled divertor only, the heating input was limited to at most 200 MJ. Maximum pulse lengths of $100 \mathrm{~s}$ were reached.

During the first week after activation of the cFIS 22 plasma discharges of total 115 were stopped by interventions of the cFIS. The response time of the cFIS is sufficient. The fast shut-off of the heating systems within $5 \mu$ s worked reliably. All interventions by the cFIS were justified. False alarms were not observed. The diagnostics for ECRH stray radiation, diamagnetic energy, and plasma density worked very reliable. The usability for the cFIS operator was satisfactory; parameter setting concept is safe and convenient.

Fig. 11 shows a regular plasma decay after end of ECRH heating. In Fig. 12 (a nearly same experiment) the radiation from the plasma got too high at $6.0 \mathrm{~s}$ and the electron temperature dropped. Unfortunately, at that time the ECE diagnosis was not available for the cFIS. There-

W7-X 20180808.028 | UTC: 15:37:10 | T0: 1533742630316689681
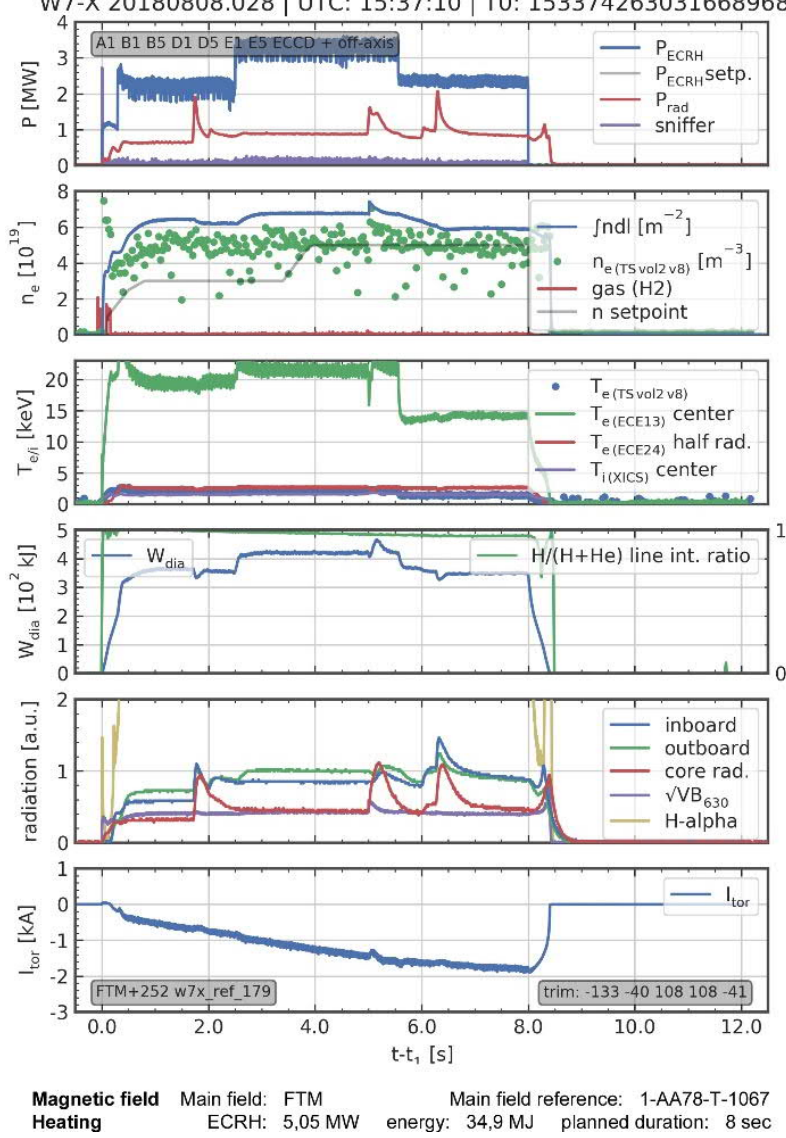

Fig. 11 Regular plasma decay after end of ECRH heating fore, the cFIS switched off the ECRH only after the diamagnetic energy also sank fast at $6.5 \mathrm{~s}$.

\section{Improvements}

The integration of the ECE-diagnostic must be checked (but it worked stand- alone properly) and some temperature drift and signal artefacts (after the discharge) at the diamagnetic loops must be eliminated.

A real-time recording of data and events in the cFIS for analysis purposes must be added, e.g. to detect jitters in signal generation.

Some timing constraints in the cFIS, which were implemented as a precaution into the first version, can be removed.

\section{Upgrade of the FIS}

The implementation of the divertor thermography is the most important of new safety-relevant diagnostics in the OP2 with a water-cooled steady state divertor (Fig. 13). Ten endoscopes that are distributed around W7-X monitor directly the surface temperature on the divertor target plates and the plasma light in the scrape off layer in front of the divertor target [10]. The optic receives infrared $(4 \mu \mathrm{m})$ and visible light $(350-900 \mathrm{~nm})$; and a fast vision software detects and classifies thermal events in each frame. The aim is to give a valid interlock signal within $<100 \mathrm{~ms}$ if the temperature exceeds a certain threshold, which can be different from target to target.
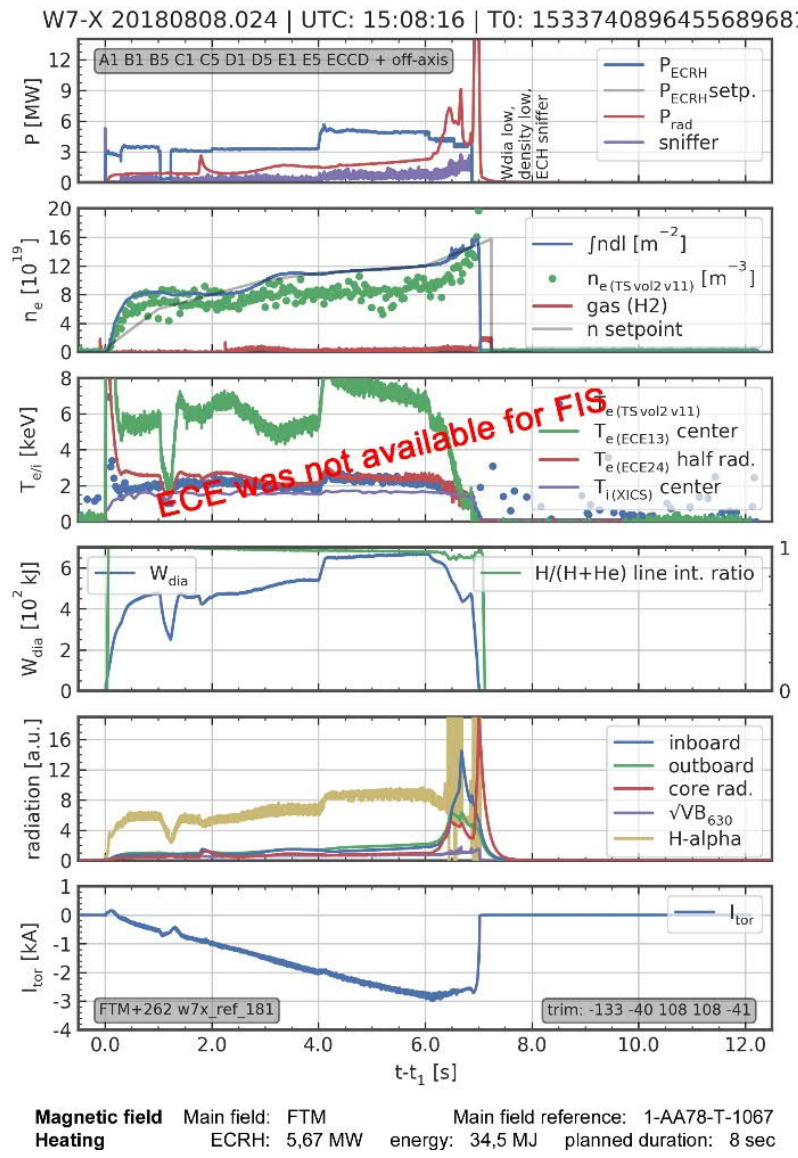

Fig. 12 Fast intervention of the cFIS induced by radiation collapse 


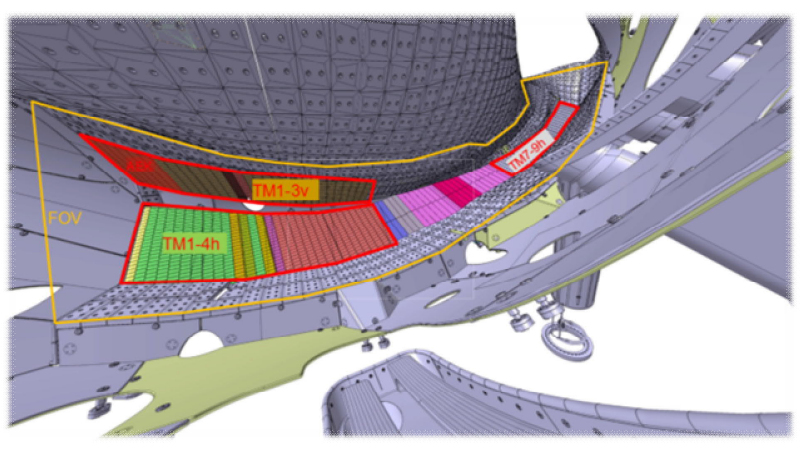

Fig. 13 Divertor thermography will observe high loaded target plates

The Ion Cyclotron Resonance Heating (ICRH) must be integrated in the system before the next operation phase.

The increasing number of heating systems and safetyrelevant diagnostics demands to cascade the used Boolean processor, or to change the system in general. Currently the eXtreme Fast Control Technology (Beckhoff) with $\mathrm{I} / \mathrm{O}$ response time $<100 \mu \mathrm{s}$ is under investigation.

The integration of a more common video diagnostic is under discussion due to the question, whether safetyrelevant data can be extracted fast enough.

\section{Summary}

The first practical experience with the central fast interlock system (cFIS) of W7 X showed that the system in principle meets all essential requirements. The structure and the realization can be completed and upgraded according to the future needs. Because of the special measuring systems, which are used, a formal fulfilment of a SIL probably will not be attainable. This shall also in future be compensated by a corresponding redundancy.

\section{Acknowledgments}

This work has been carried out within the framework of the EUROfusion Consortium and has received funding from the Euratom research and training programme 2014-2018 and 2019-2020 under grant agreement number 633053. The views and opinions expressed herein do not necessarily reflect those of the European Commission.

\section{References}

[1] Reinhard Vilbrandt, Hans-Stephan Bosch, Georg Kühner, Dirk Naujoks, Jörg Schacht, Andreas Werner, Sven Degenkolbe, Application of the engineering standard for functionnal safety to the W7-X central safety system, Fusion Eng. Des. 123 (2017) 632-636.

[2] N. Pomaro, L. Grando, A. Luchetta, F. Paolucci, F. Sartori; Design of the interlock and protection system for the SPIDER experiment, Fusion Eng. Des. 88 (2013) 980-984.

[3] A. Luchetta, N. Pomaro, C. Taliercio, M. Moressa, L. Svensson, F. Paolucci, et. al, Implementation of the SPIDER central interlock, Fusion Eng. Des. 123 (2017) 990994.

[4] D. Hartmann, B. Heinemann, R. Schroeder, P. McNeely, N. Harder, Chr. Hopf, et. al., First Time Neutral Beam Heating on Wendelstein 7-X, 46th European Physical So- ciety Conference on Plasma Physics, Milan, 2019 Poster 1076.

[5] D. Moseev, H. P. Laqua, S. Marsen, T. Stange, H. Braune, V. Erckmann., et. al., Absolute calibration of sniffer probes on Wendelstein 7-X, Rev. Sci. Instrum. 87 (2016), doi:10.1063/1.4960349 083505.

[6] K.J. Brunner, T. Akiyama, M. Hirsch, J. Knauer, P. Kornejew, B. Kursinski, et. al., Real-time dispersion interferometry for density feedback in fusion devices, J. Instrum. 13 (2018) P09002.

[7] M. Hirsch, U. Höfel, J.W. Oosterbeek, N. Chaudhary, J. Geiger, H.-J. Hartfuss, et. al., ECE Diagnostic for the initial Operation of Wendelstein 7-X, EPJ Web of Conf. 203 (2019) 3007 EC20, doi:10.1051/epjconf/201920303007.

[8] M. Endler, B. Brucker, V. Bykov, A. Cardella, A. Carls, F. Dobmeier, et. al., Engineering design for the magnetic diagnostics of Wendelstein 7-X, Fusion Eng. Des. 100 (2015) 468-494.

[9] K. Rahbarnia et al, Diamagnetic energy measurement during the first operational phase at the Wendelstein 7-X stellarator, Nucl. Fusion 58 (2018) 9.

[10] M. Jakubowski, P. Drewelow, J. Fellinger, A. Puig Sitjes, G. Wurden, A. Ali, et. al., Infrared imaging systems for wall protection in the W7-X stellarator, Rev. Sci. Instrum. 89 (2018), doi:10.1063/1.5038634 10E116. 